NASA Technical Memorandum 106265

AIAA-93-3766

\title{
Application of Controller Partitioning Optimization Procedure to Integrated Flight/Propulsion Control Design for a STOVL Aircraft
}

Sanjay Garg

Lewis Research Center

Cleveland, Ohio

and

Phillip H. Schmidt

University of Akron

Akron, Ohio

Prepared for the

1993 AIAA Guidance, Navigation, and Control Conference

sponsored by the American Institute of Aeronautics and Astronautics

Monterey, California, August 9-11, 1993

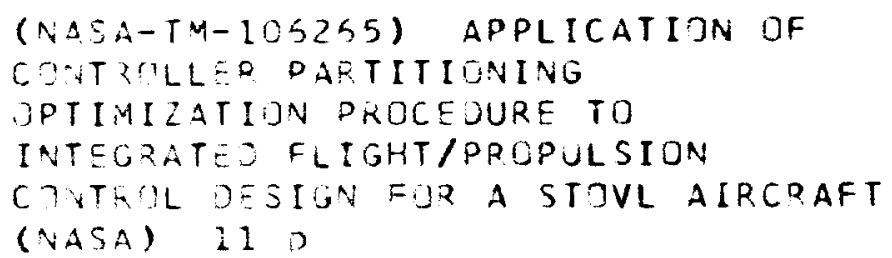

$N 94-11251$

Unclas 



\author{
Sanjay Garg* \\ NASA Lewis Research Center \\ Cleveland, $\mathrm{OH} 44135$ \\ Phillip H. Schmidt"* \\ University of Akron \\ Akron, $\mathrm{OH} 44325$
}

\begin{abstract}
A parameter optimization framework has earlier been developed to solve the problem of partitioning a centralized controller into a decentralized, hierarchical structure suitable for integrated flight/propulsion control implementation. This paper presents results from the application of the controller partitioning optimization procedure to IFPC design for a Short Take-Off and Vertical Landing (STOVL) aircraft in transition flight. The controller partitioning problem and the parameter optimization algorithm are briefly described. Insight is provided into choosing various "user" selected parameters in the optimization cost function such that the resulting optimized subcontrollers will meet the characteristics of the centralized controller that are crucial to achieving the desired closed-loop performance and robustness, while maintaining the desired subcontroller structure constraints that are crucial for IFPC implementation. The optimization procedure is shown to improve upon the initial partitioned subcontrollers and lead to performance comparable to that achieved with the centralized controller. This application also provides insight into the issues that should be addressed at the centralized control design level in order to obtain implementable partitioned subcontrollers.
\end{abstract}

\section{Introduction}

Large interconnected systems often exhibit significant coupling between the various subsystems thus requiring an integrated approach to controller design. Short Take-Off and Vertical Landing (STOVL) aircraft are an example of such subsystems. In STOVL aircraft, the forces and moments generated by the propulsion system provide control and maneuvering capabilities for the aircraft at low speeds thus creating the need for Integrated Flight/Propulsion Control (IFPC) system design. An approach to IFPC design [1] is to design a centralized controller considering the integrated airframe and propulsion system with all its interconnections as the design plant. Although such an approach yields an "optimal" design since it accounts for all subsystem

\footnotetext{
Aerospace Engineer, Advanced Controls Technology Branch. Associate Fellow AlAA.

"Professor, Dept. of Mathematical Sciences
}

interactions, it results in a high-order controller which is difficult to implement and validate. In aircraft design, it is the responsibility of the engine manufacturer to ensure that the propulsion system will provide the desired performance when installed in the airframe. The engine manufacturer therefore needs a separate engine controller to be able to independently perform extensive testing to assure adequate performance and integrity of the propulsion system in the presence of operational and safety limits. This requirement imposes the need for decentralized implementation of IFPC systems. One approach to direct decentralized design of IFPC systems is presented in Ref. [2]. This approach consists of "partitioning" the overall system into loosely coupled subsystems and then performing a decentralized control design considering one subsystem at a time. Although such an approach results in low-order, independently implementable subsystem controllers (referred to as "subcontrollers"), it has the disadvantage that it does not easily account for all the interactions between various subsystems.

An approach to IFPC design which combines the "best" aspects of the centralized and decentralized approaches is presented in Ref. [3]. This approach consists of first designing a centralized controller considering the airframe and propulsion systems as one integrated system, and then partitioning the centralized controller into decentralized airframe and engine subcontrollers with a specified interconnection structure. Here, "partitioning" means the process of approximating the high order centralized controller with two or more lower order subcontrollers, with a specified coupling structure, such that the closed-loop performance and robustness characteristics of the centralized controller are matched by the partitioned subcontrollers. A meaningful trade-off between subcontroller complexity and achievable performance for the integrated system can be performed by evaluating various controller partitionings of different levels of complexity against the performance baseline established with the centralized controller.

The most suitable decentralized control structure for IFPC systems is hierarchical with the airframe (flight) controller generating commands for the aerodynamics control surfaces as well as for the propulsion subsystem. This hierarchical structure will be discussed in the next section. A stepwise approach to determining partitioned 
subcontrollers with the decentralized hierarchical structure from a centralized IFPC design is presented in Ref. [4]. The procedure of Ref. [4], however, is ad-hoc in nature and can result in unacceptable degradation in closed-loop performance and robustness from that obtained with the centralized controller. A parameter optimization framework which can be effectively used to improve upon the subcontrollers obtained by the procedure of Ref. [4] so as to "more closely" match the centralized controller closed-loop performance and robustness characteristics was discussed in Ref. [5]. The application of the partitioned subcontroller parameter optimization algorithm to IFPC design for a Short Take-Off and Landing (STOL) aircraft was also presented in Ref. [5]. The results obtained for the STOL aircraft application demonstrated the potential of the parameter optimization approach to obtain partitioned subcontrollers that match the performance and robustness characteristics of the centralized controller while maintaining the desired subcontroller structure. The objective of this paper is to present the results from application of the controller partitioning parameter optimization procedure to IFPC design for a STOVL aircraft. The STOVL aircraft problem presents a significant increment in complexity over the STOL example studied earlier. Extensive insight is to be gained about the challenges associated with partitioning a complex IFPC centralized controller by application to the STOVL example.

The paper is organized as follows. The controller partitioning problem and the optimization framework is first briefly described. The STOVL vehicle model and the centralized as well as partitioned IFPC controller structure is then described along with a brief discussion of the performance results obtained with the initial partitioned controllers derived by using the procedure described in Ref. [4]. The results from application of the parameter optimization procedure are then presented and performance comparisons are made between the centralized controller, initial partitioned subcontrollers and the optimized subcontrollers. Issues related to the structure of the propulsion subsystem controller for IFPC implementation are then discussed in light of the results obtained by the parameter optimization procedure.

\section{Controller Partitioning:}

Problem and Optimization Algorithm

The decentralized, hierarchical controller partitioning structure is shown in Fig. 1 where the subscripts and superscripts " $a$ " and " $\mathrm{e}$ " refer to airframe and propulsion system (engine) quantities, respectively, subscript " $c$ " refers to commands, and the variables $\bar{z}$ are the controlled outputs of interest with $\bar{e}$ being the corresponding errors. The intermediate variables $\bar{z}_{\text {ea }}$ represent propulsion system quantities that affect the airframe, for example propulsion system generated forces and moments.

be stated as follows:
Given: A centralized controller K(s) s.t.

$$
\begin{gathered}
\bar{u}(s)=K(s)\left[\begin{array}{l}
\bar{e}(s) \\
\bar{y}(s)
\end{array}\right], \\
\text { where } \bar{u}=\left[\begin{array}{l}
\bar{u}_{k} \\
\bar{u}_{c}
\end{array}\right], \bar{e}=\left[\begin{array}{l}
\bar{e}_{\bar{e}} \\
\bar{e}_{e}
\end{array}\right] \text {, and } \bar{y}=\left[\begin{array}{l}
\bar{y}_{e} \\
\bar{y}_{e}
\end{array}\right],
\end{gathered}
$$

and a particular set of the interface variables $\overline{\mathbf{z}}_{\boldsymbol{c}}$,

Find: Decentralized airframe and engine subcontrollers, $K^{*}(s)$ and $K^{e}(s)$, respectively, with

$$
\left[\begin{array}{l}
\bar{u}_{a}(s) \\
\bar{z}_{e s}(s)
\end{array}\right]=K^{s}(s)\left[\begin{array}{l}
\bar{e}_{a}(s) \\
\bar{y}_{\mathrm{a}}(s)
\end{array}\right] \text {, and } \bar{u}_{e}(s)=K^{e}(s)\left[\begin{array}{l}
\bar{e}_{e a}(s) \\
\bar{e}_{e}(s) \\
\bar{y}_{e}(s)
\end{array}\right](2)
$$

So that: The closed-loop performance and robustness with the subcontrollers $K^{\prime \prime}(s)$ and $K^{e}(s)$ match those with the centralized controller $K(s)$ to a desired accuracy. Furthermore, the engine subcontroller $K^{e}(s)$ should have the structure of a command tracking controller for the interface variables $\bar{z}_{e}$ to allow for independent checkout of the propulsion system.

The approach for solving the controller partitioning problem, as discussed in Ref. [5], consists of optimization of a suitable cost function over the state-space parameters of the partitioned subcontrollers using an analytical expression for the gradient of the cost function. The initial partitioned subcontrollers to start the parameter optimization are obtained using the procedure discussed in Ref. [4] and the cost function to be minimized is chosen to be of the form

$$
\mathrm{J}(\overline{\mathrm{p}})=\mathrm{J}_{\text {per }}(\overline{\mathrm{p}})+\mathrm{J}_{\text {tack }}(\overline{\mathrm{p}})
$$

where $\bar{p}$ is the vector of parameters over which the optimization takes place, $J_{\text {perr }}(\bar{p})$ reflects the performance requirements (including robustness) and $J_{\text {tmat }}(\bar{p})$ reflects the $\bar{z}_{\text {ea }}$ tracking requirement. The performance cost is chosen to be

$J_{p e r t}(\bar{p})=\int_{\omega_{1}}^{\omega_{0}} \sum_{k}\left(\sigma_{k}\left[W_{1}(j \omega) \cdot(K(j \omega)-\tilde{K}(\bar{p})(j \omega)) \cdot W_{0}(j \omega)\right]\right)^{2} d \omega$

where $\sigma_{k}[\cdot]$ denotes the $k^{\text {th }}$ singular value of a matrix, and $\tilde{\mathrm{K}}(\mathrm{s})$ is the "equivalent" centralized controller obtained by assembling the partitioned subcontrollers. Plant information consisting of the state-space representation of the transfer function matrices $G(s)$ and $G_{e}$ (s), defined by 


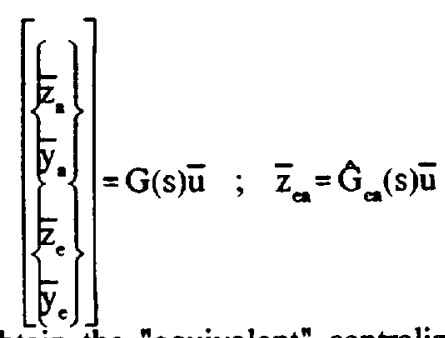

is used to obtain the "equivalent" centralized controller, $\tilde{\mathbf{K}}$ (s), as described in Ref. [5]. The above choice of $\mathrm{J}_{\text {perr }}(\overline{\mathrm{p}})$ corresponds to the $\mathrm{H}_{2}$ norm of the weighted difference between the designed centralized controller and the "equivalent" centralized controller. The frequency band, $\left[\omega_{1}, \omega_{2}\right]$ is the interval over which a good match between the two controller transfer function matrices is sought while the input and output frequency weightings, $W_{i}(s)$ and $W_{n}(s)$, respectively, allow for emphasizing certain frequency ranges and directions in obtaining this good approximation.

The $\bar{z}_{\text {ea }}$ tracking cost $\mathrm{J}_{\text {trox }}(\overline{\mathrm{p}})$ is chosen to be

$$
J_{\text {tack }}(\bar{p})=\int_{\omega_{1}}^{\omega_{i}} \sum_{i} \lambda_{i} \cdot\left(\mathbf{T}_{\text {ceat }}^{i}(j \omega)-\hat{T}^{\prime}(\overline{\mathbf{p}})(j \omega) \boldsymbol{\|}_{2}\right)^{2} d \omega
$$

where: $T_{\text {remt }}^{1}(s)$ is the transfer function vector from the airframe commands $\bar{z}_{a}$ to the $i^{i \text { it }}$ interface variable $z_{a}{ }^{1}$ with the centralized controller, $\hat{T}^{i}(s)$ is the transfer function vector from the airframe commands $\bar{z}_{\text {a }}$ to the $i^{\text {ts }}$ commanded interface variable, $z_{\text {e }}^{\prime}$, with the partitioned airframe controller; $\lambda_{i}$ is a weighting which could be chosen to be frequency dependent; and $\boldsymbol{H}_{2}$ denotes the Euclidean norm of a row vector. Note that for the partitioned subcontrollers to closely match the performance achieved with the centralized controller, the response of the interface variables $\bar{z}_{a}$ to airframe commands $\bar{z}_{a}$, with the partitioned subcontrollers must match the corresponding response with the centralized controller because the interface variables (e.g., propulsion system generated thrusts) significantly affect the airframe responses $\bar{z}_{\mathrm{a}}$. Thus requiring $T^{i}{ }_{\text {eemt }}$ (s) to closely match $\hat{T}^{\prime}(s)$, as reflected in $J_{\text {lack }}(\bar{p})$, will result in partitioned controllers such that $z i$ appears to be a command for $z^{i}$. The weights $\lambda_{1}$ allow the control designer to provide relative weighting for enforcing the command tracking structure among the various elements of $z_{c a}^{i}$ and for trading off the performance cost against the cost of enforcing this command tracking structure.

In Ref. [5], a procedure was presented for developing the analytical gradients of the cost functions described in (3) and (5) with the parameter vector $\bar{p}$ consisting of the matrix elements in the state-space realization of the subcontroller transfer function matrices $K^{\mathbf{a}}(s)$ and $K^{e}(s)$. Based on these analytical expressions for the gradients, a numerical algorithm for minimizing the cost $J(\bar{p})$ was developed. This algorithm is shown in flowchart form in Fig. 2 and is briefly described in the following.

The fixed data used by the algorithm of Ref. [5] are state-space representations for the plant system matrices $G(s)$ and $\hat{G}_{e a}$ (s) (as defined in (4)), the centralized controller $K(s)$, the weighting matrices $W_{1}(s)$ and $W_{0}(s)$, the weights $\lambda_{b}$, and the partitioning structure consisting of subcontroller inputs and outputs and the interface variables. The initial partitioning is obtained using the stepwise procedure described in Ref. [4] or as a result of order reduction on an earlier optimized partitioning. This initial partitioning is converted to a "minimal parameter" form described in Ref. [5] and an initial parameter vector is generated. For a given parameter vector, the cost and the analytical expression based cost gradients are then calculated. The Broyden-Fletcher-Goldfarb-Shanno method (see Ref. [6]) for search direction and linesearch is used to update the parameter vector such that both the cost function and the norm of the gradient vector is reduced. This linesearch is constrained so as to maintain the stability of the subcontrollers. Various convergence criteria involving reduction in the cost function between successive parameter updates, the norm of the difference between previous and updated parameter vector, and the norm of the gradient vector are used to check for "optimality". The output of this procedure is a set of partitioned subcontrollers which can be analyzed for performance and stability robustness. If these are unacceptable, then the optimization can be continued with either tighter convergence criteria or modified choice of the weighting matrices $W_{1}(s)$ and $W_{0}(s)$ and/or the weights $\lambda_{1}$.

\section{STOVL Aircraft Model and}

\section{Initial Controller Partitioning}

The controller partitioning optimization procedure discussed above was applied to the IFPC design for a STOVL aircraft in the decelerating transition during approach to hover landing flight phase. A schematic diagram of the aircraft is shown in Fig. 3. The aircraft is powered by a two-spool turbofan engine and is equipped with the following control effectors: left and right elevons used collectively as elevator and differentially as ailerons; rudder, ejectors to provide propulsive lift at low speeds and hover, a 2D-CD (two dimensional convergentdivergent) vectoring aft nozzle; a vectoring ventral nozzle for pitch control and lift augmentation during transition; and jet reaction control systems (RCS) for pitch, roll and yaw control during transition and hover. Engine compressor bleed flow (WB3) is used for the RCS thrusters and the mixed engine flow is used as the primary ejector flow. The aircraft and engine model and the design of the centralized controller for a linear integrated design model are discussed in detail in Refs. [7,8]. The centralized controller was partitioned into decoupled lateral and longitudinal-plus-engine subcontrollers as discussed in Ref. [8], and the longitudinal-plus-engine controller was further partitioned into separate airframe and engine 
subcontrollers which have the decentralized, hierarchical structure of Fig. 1. In the following, the vehicle model is first summarized, and the performance results with the initial partitioned subcontrollers obtained from Ref. [4] are briefly discussed.

The linear integrated aircraft longitudinal dynamics and engine dynamics small perturbation model is of the form

where the state vector is

$$
\dot{\bar{x}}=A \bar{x}+B \bar{u}
$$

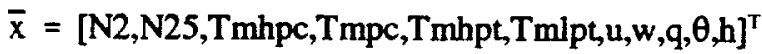

$$
\begin{aligned}
& \text { with } \\
& \text { N2 = Engine Fan Speed, rpm } \\
& \text { N25 = High Pressure Compressor Speed, rpm } \\
& \text { Tmhpc = High Press. Compressor Metal Temp., }{ }^{\circ} \mathrm{R} \\
& \text { Tmpc = Burner Metal Temp., }{ }^{\circ} \mathrm{R} \\
& \text { Tmhpt }=\text { High Pressure Turbine Metal Temp., }{ }^{\circ} \mathrm{R} \\
& \mathrm{Tm} \text { ipt }=\text { Low Pressure Turbine Metal Temp., }{ }^{\circ} \mathrm{R} \text {. } \\
& \mathrm{u}=\text { Axial Velocity, } \mathrm{ft} / \mathrm{s} \\
& \mathrm{w}=\text { Vertical Velocity, } \mathrm{ft} / \mathrm{s} \\
& \mathrm{q} \quad=\text { Pitch Rate, } \mathrm{rad} / \mathrm{s} \\
& \theta=\text { Pitch Attitude, rad } \\
& \mathrm{h}=\text { Altitude, } \mathrm{ft}
\end{aligned}
$$

The control inputs partitioned into airframe and engine control inputs are

with

$$
\begin{gathered}
\overline{\mathrm{u}}_{\mathrm{a}}=[\mathrm{\delta e}, \mathrm{AQR}, \mathrm{ANG} 79, \mathrm{ANG}]^{\mathrm{T}} \\
\overline{\mathrm{u}}_{\mathrm{e}}=[\mathrm{WF}, \mathrm{A} 8, \mathrm{ETA}, \mathrm{A} 78]^{\mathrm{T}}
\end{gathered}
$$

$$
\begin{array}{ll}
\delta \mathrm{e} & =\text { Elevator Deflection, deg } \\
\mathrm{AQR} & =\text { Pitch } \mathrm{RCS} \text { Area, in }
\end{array}
$$$$
\text { ANG79 = Ventral Nozzle Vectoring Angle, deg }
$$$$
\text { ANG8 = Aft Nozzle Vectoring Angle, deg }
$$$$
\text { WF } \quad \text { Fuel Flow Rate, lbm/hr }
$$$$
\text { A8 = Aft Nozzle Area, in }{ }^{2}
$$$$
\text { ETA = Ejector Butterfly Angle, deg }
$$

A78 = Ventral Nozzle Area, in ${ }^{2}$

The controlled outputs for the airframe and engine systems are

$$
\bar{z}_{\mathrm{a}}=[\mathrm{Vv}, \mathrm{Qv}, \gamma]^{\top} ; \overline{\mathrm{z}}_{\mathrm{e}}=\mathrm{N} 2
$$

$$
\begin{gathered}
\text { where } \begin{aligned}
\mathrm{V} & =\dot{\mathrm{V}}+0.1 \mathrm{~V}, \mathrm{Qv}=\mathrm{q}+0.3 \theta \text { with } \\
& =\text { True Airspeed, } \mathrm{ft} / \mathrm{s} \\
\dot{\mathrm{V}} & =\text { Acceleration Along Flight Path, } \mathrm{ft} / \mathrm{s}^{2} \\
\gamma & =\text { Flight Path Angle, } \mathrm{deg}
\end{aligned}
\end{gathered}
$$

and the other outputs as discussed under state description with units of $q$ and $\theta$ in degrees. As discussed in Ref. [7], the above choice of $\bar{z}_{\mathrm{a}}$ corresponds to providing the pilot with an acceleration command, velocity hold system in the forward axis; pitch rate command, attitude hold system in the pitch axis; and direct command of the flight path angle for vertical axis control. The choice of $\bar{z}_{e}$ allows for setting the engine operating point independent of the aircraft maneuver.
The inputs to the airframe and the engine controllers are the tracking errors $\bar{e}_{\mathrm{a}}$ and $\bar{e}_{e}$ corresponding to $\bar{z}_{a}$ and $\bar{z}_{e}$ respectively, and the measurement feedbacks

$$
\bar{y}_{\mathrm{a}}=[\mathrm{V}, \dot{\mathrm{V}}, \theta, \mathrm{q}, \mathrm{y}]^{\mathrm{T}} ; \overline{\mathrm{y}}_{\mathrm{c}}=[\mathrm{N} 2, \mathrm{WB} 3]^{\mathrm{T}}
$$

where WB3 is the compressor bleed flow demanded by the RCS control.

The interface from the propulsion system model to the airframe model is defined by the gross thrusts from the three engine nozzle systems, i.e.

$$
\begin{aligned}
& \text { where } \\
& \text { FG9 = Aft Nozzle Gross Thrust, lbf } \\
& \text { FGE = Ejector Gross Thrust, lbf } \\
& \text { FGV = Ventral Nozzle Gross Thrust, lbf. }
\end{aligned}
$$$$
\bar{z}_{e}=[F G 9, F G E, F G]^{T}
$$

Using the procedure described in Ref. [4], an initial partitioning of the centralized longitudinal-plus-engine controller into separate airframe and engine subcontrollers was obtained. These initial partitioned subcontrollers provided close matching of the partitioned closed-loop system response to the centralized closed-loop system response for aircraft velocity and flight path commands $\left(\mathrm{Vv}_{\mathrm{c}}\right.$ and $\left.\gamma_{c}\right)$ and the engine fan speed command $\left(\mathrm{N} 2{ }_{c}\right)$. However, this partitioned system response to the pitch variable command $\left(Q v_{c}\right)$ showed significant deviation from the corresponding response for the centralized system. Shown in Fig. 4 is the closed-loop response of the system with the centralized and initial partitioned subcontrollers for an example pitch variable command. Although the level of decoupling in the $V v, \gamma$ and $N 2$ response to $Q v_{r}$ with the initial partitioned subcontrollers is comparable to that obtained with the centralized controller, there is significant degradation in the tracking of the Qv command itself. The partitioning parameter optimization algorithm was applied to this example to investigate whether the response to pitch variable command can be improved.

\section{Controller Partitioning Optimization}

As mentioned earlier, the controller partitioning parameter optimization algorithm was earlier applied to a linear model of a STOL (Short Take-Off and Landing) aircraft and the results were presented in Ref. [5]. The STOVL problem being addressed in this paper is considerably more complex than the STOL example in that the centralized controller is not square $(K(s)$ has dimension 8 by 10 for the STOVL problem as opposed to 4 by 4 for the STOL example), and there are 3 interface variables $\left(\bar{z}_{z a}\right)$ as opposed to just one for the STOL example. Further insight is to be gained into the suitability of the controller partitioning parameter optimization procedure and the proper selection of the various weighting factors by applying the procedure to this complex STOVL example. In the following, the issues related to the proper choice of weighting factors are first discussed and then the results are presented for a set of optimized subcontrollers. 
Choice of Weighting Factors in the Optimization Cost

Initially, the weighting matrices for the performance cost function $J_{\text {perr }}(\bar{p})$ were chosen to be consistent with those that led to the successful results for the STOL example, i.e., $W_{1}=I$, and $W_{0}(s)=G(s) \cdot[1+K(s) G(s)]^{-1}$, where $I$ is an appropriately dimensioned identity matrix and $G(s)$ is the plant system matrix defined earlier. This choice of weighting then corresponds to minimizing the $\mathrm{H}_{2}$ norm of

$$
(K(j \omega)-\tilde{K}(\bar{p})(j \omega)) G(j \omega) \cdot[I+K(j \omega) G(j \omega)]^{-1}
$$

which is the frequency weighted loop transfer matrix error at the control inputs $\bar{u}$. The weights $\lambda_{i}$ in $J_{\text {tack }}(\bar{p})$ were chosen to be

$$
\lambda_{1}=\left[\left|T_{\text {cent }}^{i}(j \omega)\right|_{m=0} l_{2}\right]^{-1}
$$

which corresponds to normalization of the tracking error for $i^{\text {th }}$ interface variable by the euclidean norm of the steady-state response of the $i^{\text {th }}$ interface variable to the airframe commands $\left(\bar{z}_{2}\right)$ for the centralized system.

Exercising the optimization algorithm with the above choice of weightings resulted in significant reduction of the total cost, $J(\bar{p})$, as well as reduction in both the individual elements of the cost, $J_{\text {perr }}(\bar{p})$ and $J_{\text {tack }}(\bar{p})$. However, when the closed-loop system was analyzed with the optimized partitioned subcontrollers, the performance was much degraded over the initial partitioning. Note that the choice of $\mathrm{W}_{0}(\mathrm{~s})$ corresponding to (7) was driven by the small gain theorem according to which the closed-loop system with the partitioned subcontrollers will remain stable if $\mathbf{I}(\mathrm{K}(j \omega)-\tilde{K}(\overline{\mathbf{p}})(j \omega)) \mathrm{G}(j \omega) \cdot[1+K(j \omega) \mathrm{G}(j \omega)]^{-1} \|_{\infty}<1$, where $\mid \cdot \|_{\infty}$ denotes the $H_{\infty}$ norm. This type of weighting is used in controller order reduction problems (see Ref. [9]) and was successful for the STOL controller partitioning example because the controller and the plant were square. For a square system, matching the loop transfer function matrix at the control inputs $(\overline{\mathbf{u}})$ will in general imply a good match with the loop transfer function matrix at the controlled outputs $(\bar{z})$, which corresponds to matching closed-loop performance. This sort of relationship does not necessarily hold true for a nonsquare system as was experienced for the STOVL example.

Next, the weights in the performance cost, $J_{\text {pern }}(\bar{p})$, were modified such that $W_{i}(s)=G_{z}(s)$ and $W_{0}(s)=I$, where $G_{z}(s)$ is defined such that $\bar{z}=G_{z}(s) \bar{u}$. This choice of weighting corresponds to minimizing the $\mathrm{H}_{2}$ norm of

$$
G_{z}(j \omega)(K(j \omega)-\tilde{K}(j \omega))
$$

which is the loop transfer matrix error at the controlled outputs $\bar{z}$. The "optimal" partitioned subcontrollers obtained by exercising the optimization algorithm with these modified weightings were such that these led to improved closed-loop performance over the initial partitioned subcontrollers in terms of more closely matching the decoupled command tracking properties of the system with the centralized controller. However, these optimized subcontrollers had excessive control and control rate requirements which will result in significant system performance degradation when implementing the subcontrollers with control actuation and rate limits. Furthermore, the required interface variable $\left(\bar{z}_{\text {cas }}\right)$ command tracking structure for the engine subcontroller was not retained by these optimized subcontrollers.

Detailed analyses of the above results revealed that the equivalent controller $\widetilde{K}(s)$ obtained by assembling these optimized subcontrollers has totally different input/output response characteristics as compared to the designed centralized controller $K(s)$. Note that for the STOVL example there are only four controlled variables, $\bar{z}$, whereas there are eight control inputs, $\bar{u}$. Due to this redundancy in control effectors, theoretically there are an infinite number of solutions for a control law which matches the loop transfer function matrix at the controlled outputs with the designed centralized controller. Without any constraints on the control usage, "larger" control provides "better" matching of the loop transfer function matrix, and so the parameter optimization algorithm tends to a solution for $\tilde{\mathrm{K}}(\mathrm{s})$ which has excessive control requirements. Further note that the formulation for the "tracking" cost, $\mathrm{J}_{\text {trax }}(\overline{\mathrm{p}})$, was based on the hypothesis that "for the partitioned subcontrollers to closely match the performance achieved with the centralized controller, the response of the interface variables $\bar{z}_{a}$ to airframe commands $\bar{z}_{\mathrm{a}}$ with the partitioned subcontrollers must match the corresponding response with the centralized controller". This hypothesis is no longer true, for the above choice of weightings in $\mathrm{J}_{\text {purn }}$ due to the controller redundancy effect discussed earlier. Although the choice of $J_{\text {tack }}$ forces the $\bar{z}_{e}$, response to $\bar{z}_{\mathrm{a}}$ command input with the optimized partitioned subcontrollers to match the $\bar{z}_{\text {ca }}$ response with the centralized controller, the $\bar{z}_{\text {ea }}$ response with the partitioned subcontrollers is itself very different from the corresponding response with the centralized controller. Thus, for the optimized partitioned subcontrollers the $\bar{z}_{e a}$ and $\bar{z}_{a}$ responses are very different from each other and the desired command tracking relationship between $\bar{z}_{e,}$ and $\bar{z}_{e}$ is not maintained.

In order to overcome the problems due to control redundancy and to force the "same" control solution for the optimized subcontrollers as for the centralized controller, the optimization was performed with $W_{1}(s)$ and $\mathrm{W}_{\mathrm{o}}(\mathrm{s})$ chosen to be appropriately dimensioned identity matrices, i.e. minimize the $\mathrm{H}_{2}$ norm of the unweighted controller approximation error

$$
(K(j \omega)-\tilde{K}(j \omega)) \text {. }
$$

With this choice of $J_{\text {pert }}$ the optimized subcontrollers showed improvements in all areas in which there were problems with the previous formulations of $J_{\text {perr }}$, however the resulting engine subcontroller was very high bandwidth 
with virtually flat $z^{\prime} \rightarrow z^{i}$ response across a large frequency band for the closed-loop engine subsystem. The parameter vector over which the optimization was performed included elements of the direct feedthrough matrix from the error in interface variable command tracking $\left(\overline{\mathrm{e}}_{e}=\overline{\mathrm{z}}_{\mathrm{e}}-\overline{\mathrm{z}}_{\mathrm{e}}\right)$ to the engine control inputs $\left(\overline{\mathrm{u}}_{\mathrm{e}}\right)$ for the engine subcontroller. As the optimization proceeded, these elements tended to become large thus implying a large bandwidth for the interface variable command tracking portion of the engine subcontroller. In order to limit the engine subcontroller bandwidth and provide adequate control gain attenuation, the engine subcontroller structure was modified to keep this direct feedthrough matrix zero for the partitioning optimization. To provide further frequency roll-off, the weights $\lambda_{1}$ in $J_{\text {inct }}$ were modified to be

$$
\lambda_{i}=\frac{10}{(j \omega+10)} \cdot\left[\left|T T_{\text {cent }}^{i}(j \omega)\right|_{\omega=0} I_{2}\right]^{-1}
$$

The results with this choice of weighting factors in $J_{\text {perf }}$ and $J_{\text {ladi }}$ are discussed in the following subsection.

\section{Optimization Results and Discussion}

Shown in Fig. 5 are the maximum singular values $(\bar{\sigma})$ of three controller partitioning error measures corresponding to $(10),(9)$ and (7) for the initial and the optimized partitioned subcontrollers. The optimized partitioning shows improvement over the initial partitioning for all three measures although $\mathbf{J}_{\text {perf }}$ corresponded to only minimizing $\mathrm{H}_{2}$ norm of (10). When the optimization was done with $\mathrm{J}_{\text {per }}$ corresponding to either (7) or (9), the other two error measures $((10)$ and (9) or (7), respectively) showed an increase over a significant frequency region leading to the problems discussed earlier. The significant reduction in the unweighted controller approximation error $(\bar{\sigma}[K(j \omega)-\bar{K}(j \omega)])$ shows that the optimized partitioned subcontrollers better match the centralized controller, the significantly decreased error in the loop transfer function matrix at the controlled outputs ( $\bar{\sigma}\left[\mathrm{G}_{z}(j \omega)(\mathrm{K}(j \omega)-\overline{\mathrm{K}}(\mathrm{j} \omega))\right]$ ) indicates that the optimized subcontrollers will better match the decoupled command tracking properties of the centralized controller, and the reduced error in the weighted loop transfer function matrix at the control inputs $\left(\bar{\sigma}\left[(K(j \omega)-\tilde{K}(j \omega)) G(j \omega)[I+K(j \omega) G(j \omega)]^{-1}\right]\right)$ indicates that the optimized subcontrollers will better match the stability robustness characteristics of the centralized controller with respect to uncertainties at the control inputs. Note that for the optimized subcontrollers, $\mid(K-\bar{K}) G[I+K G]^{-1} I_{\infty}<1$, which guarantees that the closed-loop system will be stable with the optimized partitioned subcontrollers.

The closed-loop response to pitch variable command for the optimized partitioned subcontrollers is shown in Fig. 4. The optimized partitioned subcontrollers provide the desired improvement over the initial partitioning in the Qv command tracking response while the level of decoupling in the $\mathrm{Vv}, \gamma$ and $\mathrm{N} 2$ responses is maintained. For all the other command inputs $\left(\bar{z}_{c}\right)$ also, the optimized subcontrollers provided equally good or slightly improved performance as compared to the initial partitioned subcontrollers. The control input $(\bar{u})$ requirements for all commands with the optimized subcontrollers were quite similar to those with the centralized controller.

The results in Fig. 6 show the effect of $\mathrm{J}_{\text {tmack }}$ in imposing the engine subcontroller command tracking structure discussed earlier. Shown in Fig. 6 are the frequency response magnitude plots for the three $z^{1} \rightarrow z^{i}$ responses with the initial and optimized engine subcontroller. The optimization results in increased tracking bandwidth for aft nozzle and ejector thrust commands ( $\mathrm{FG} 9_{c}$ and $\mathrm{FGE}_{c}$ ), but a decrease in the tracking bandwidth for ventral nozzle command $\left(\mathrm{FGV}_{\mathrm{c}}\right.$ ) accompanied with a somewhat increased steady-state tracking error. Also, although not shown here, there was significant coupling in the FGV response from the FG9 and FGE commands indicating that this optimized subcontroller will not meet the requirements for independent check-out of the propulsion subsystem. This shortcoming of the optimized engine subcontroller can be overcome by varying the weightings $\lambda_{i}$ in $J_{\text {trak }}$ as was successfully demonstrated in Ref. [5] for the STOL example. However, this was not done for the current STOVL example because experience gained by exercising the partitioning optimization algorithm on this complex problem suggests a modification in the problem formulation which will be discussed next.

Consider the engine subcontroller as consisting of two components:

$$
\bar{u}(s)=K_{e}^{e}(s) \bar{e}_{e}(s)+K_{e}^{e}(s)\left[\begin{array}{c}
\bar{e}_{e}(s) \\
\bar{y}_{e}(s)
\end{array}\right]
$$

The centralized controller, $K(s)$, contains a sub-block corresponding to the same inputs and outputs as $K_{e}^{*}(s)$, the $\bar{z}_{e}$ command tracking and feedback augmentation portion of the engine subcontroller. The choice of $J_{\text {perf }}$ directly provides a "design" constraint on $K_{e}^{e}(s)$ in terms of closely matching the equivalent portion of the centralized subcontroller. There is no such direct information available from the centralized controller regarding the design of $K_{e}^{*}(s)$, the $\bar{z}_{e}$ command tracking portion of the engine subcontroller. The centralized controller only provides guidelines on the minimum $\bar{z}_{\text {ea }}$ command tracking bandwidth required of the engine subcontroller. As discussed in Ref. [4], there are other constraints placed on the design of $\mathrm{K}_{\mathrm{e}}^{\mathrm{e}}(\mathrm{s})$ such as disturbance rejection requirements, robustness to modelling uncertainties and control actuation limits etc. For the initial controller partitioning [4], a $\mathrm{K}_{-}^{\mathrm{e}}(\mathrm{s})$ is designed such that it best meets the various design requirements. However, the $\mathrm{K}_{\text {e }}^{\mathrm{e}}$ (s) gets modified during the partitioning optimization, and since the $J_{\text {Inct }}$ formulation does not 
adequately reflect all the design requirements for $\mathrm{K}^{\mathrm{e}}(\mathrm{s})$, the optimization can result in an unacceptable $\mathrm{K}^{\mathrm{e}}$ (s). An approach to keeping the $\mathrm{K}^{\mathrm{e}}$ (s) portion of the engine subcontroller fixed during the optimization is currently being investigated.

In implementation of propulsion system control laws, the command for the engine operating point is determined from an open-loop schedule which is based on some measure of the gross thrust demanded from the propulsion system. For the STOVL example, this corresponds to the fan speed command being a function of the gross thrust commands for the three nozzles, i.e. $\mathrm{N} 2 \mathrm{c}=$ $f\left(\mathrm{FG}_{c}, \mathrm{FGE}_{c}, \mathrm{FGV}_{c}\right)$. Generalizing, this implies that the engine commands $\bar{z}_{e}$ might be a function of the interface variable commands $\bar{z}_{a}$. In order for the engine subsystem to track the interface variable commands in the presence of such an outer loop, it is necessary that the engine subcontroller be such that it provides decoupling of the interface variable response to the engine commands, i.e. $\bar{z}_{e_{1}} \rightarrow \bar{z}_{e a} \approx 0$. Shown in Fig 7 is the response of the three interface variables, FG9, FGE and FGV, to a step engine command $\left(\mathrm{N} 2_{\mathrm{c}}=200 \mathrm{rpm}\right)$ for the centralized controller, the initial partitioned subcontrollers and the optimized partitioned subcontrollers. All the quantities shown in Fig. 7 correspond to perturbations from a trim condition. The initial partitioned engine subcontroller was designed to take into account the $\bar{z}_{e_{1}} \rightarrow \bar{z}_{e} \approx 0$ requirement and this is reflected in the responses shown in Fig. 7. The $\bar{z}_{c_{c}}-\bar{z}_{c a}$ decoupling requirement was not imposed in the centralized control design because it was thought that since the gross thrusts $\left(\bar{z}_{e_{a}}\right)$ significantly affect the airframe response $\left(\bar{z}_{\mathrm{a}}\right)$, imposing the criterion of decoupled command tracking for airframe and engine commands $\left(\bar{z}_{a}\right.$ and $\left.\bar{z}_{e}\right)$ will result in the decoupling of thrust response to engine commands. However, due to the effect of control redundancy discussed earlier, the centralized controller is such that it results in significant coupling from the engine commands to the thrust response while decoupling of the airframe response is maintained by appropriate usage of the airframe control inputs $\left(\bar{u}_{a}\right)$. Since the optimized partitioned subcontrollers match the control usage of the centralized controller, via the $J_{\text {perf }}$ formulation discussed earlier, these too result in significant coupling of the thrust response to fan speed commands as seen from Fig. 7.

An approach to impose this $\bar{z}_{e_{1}} \rightarrow \bar{z}_{\text {ea }}$ decoupling requirement in the parameter optimization procedure is to add an appropriately formulated cost function to the total cost $\mathrm{J}$ to be minimized. This approach is currently being investigated. Another approach might be to directly consider this decoupling requirement at the centralized control design level. However, this latter approach will require further research in control theory as most multivariable control design techniques such as Linear Quadratic Gaussian, $\mathrm{H}_{\infty}$, etc. do not have any direct means of incorporating design criterion which corresponds to penalizing an individual input/output response.

\section{Conclusions}

Results were presented from the application of a controller partitioning parameter optimization algorithm to Integrated Flight/Propulsion Control (IFPC) design for a Short Take-Off and Vertical Landing (STOVL) aircraft. Insight was provided into the effect of various user selected weighting parameters in the optimization cost and it was shown that with an appropriate choice of these weighting parameters, partitioned subcontrollers could be obtained that closely matched the closed-loop performance and robustness characteristics of the centralized controller. However, the current optimization problem formulation was found to be inadequate in terms of meeting the requirements placed by the need to be able to independently check-out the propulsion subsystem. This requirement is primarily that the engine subcontroller have a decoupled command tracking structure for the interface variables from the engine to the airframe, i.e. engine developed thrusts. A modification to the optimization procedure, which consists of keeping a subportion of the engine subcontroller fixed during optimization, is currently being investigated. As a result of this application study, a need was identified for developing modifications to modern multivariable control design techniques which will allow for penalizing an individual closed-loop input/output response in a multivariable system.

\section{References}

[1] Smith, K.L., "Design Methuds for Integrated Control Systems," AFWAL-TR-86-2 103, Wright Patterson AFB, OH, Dec. 1986.

[2] Shaw, P.D, Rock, S.M., and Fisk, W.S., "Design Methods for Integrated Control Systems," AFWAL-TR-88-2061, Wright Patterson AFB, OH, June 1988.

[3] Garg, S., Ouzts, P J., Loreizo, C.F., and Mattern, D.L., "IMPAC An Integrated Methodology for Propulsion and Airframe Control," Proceedings of the 1991 American Control Conference, June 1991, Boston, MA, Vol 1, pp. 747-754.

[4] Garg, S.,"Partitiuning of Centralized Integratal Fligh/Propulsion Control Design for Decentralized Implementation," IEEE Transactions on Control Systems Technology, Vol. 1, No. 2, June 1993.

[5] Schmidt, P.H., Garg, S., and Holowecky, B., "A Parameter Optimization Approach to Controller Partitioning for Integrated FlightPropulsion Control," IEEE Transactions on Control Svstems Technologi, Vol. 1, No. 1, March 1993, pp. 21-36.

[6] R. Fletcher, Practical Methods of Optimization. New York: Wiley, 1987

[7] Garg, S., and Ouzts, PJ., "Robust Integrated Fight/Propulsion Control Design for a STOVL Aircraft using H-Infinity Control Design Techniques," Automatica, Vol. 29, No. 1, pp. 129-145, 1993.

[8] Garg, S., and Mattern, D.L., "Application of an Integrated FlighuPropulsion Control Design Methodnlogy to a STOVL Aircraft," AlAA Paper No. 91-2792, presented at the AlAA Guidance, Navigation and Control Conference, New Orleans, LA, August 1991.

[9] Enus, D.F., "Model Reduction for Control System Design", Ph.D. dissertation, Dept. of Aeronantics and Astronautics, Stanford University, Stanford, CA, 1984. 


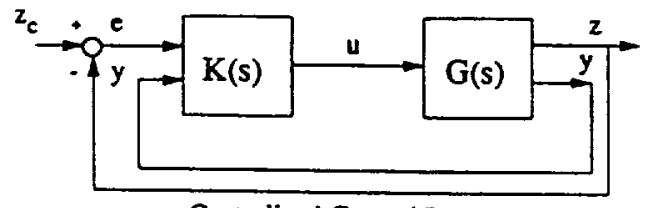

Centralized Control Loop

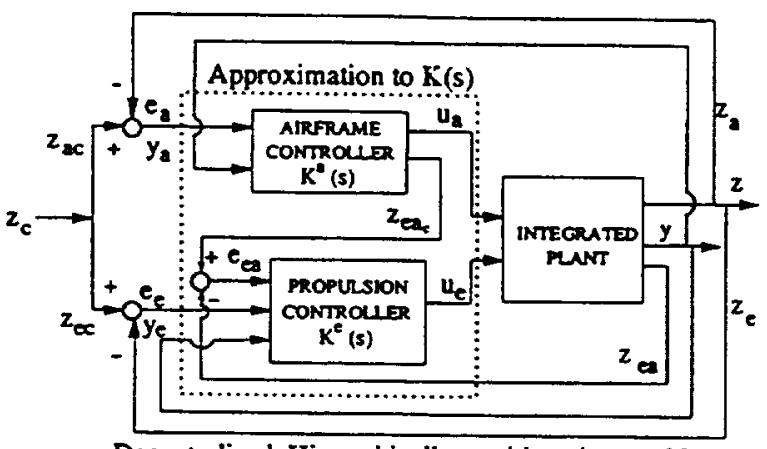

Decentralized, Hierarchically paritioned control loop

Fig. 1 Controller partitioning structure
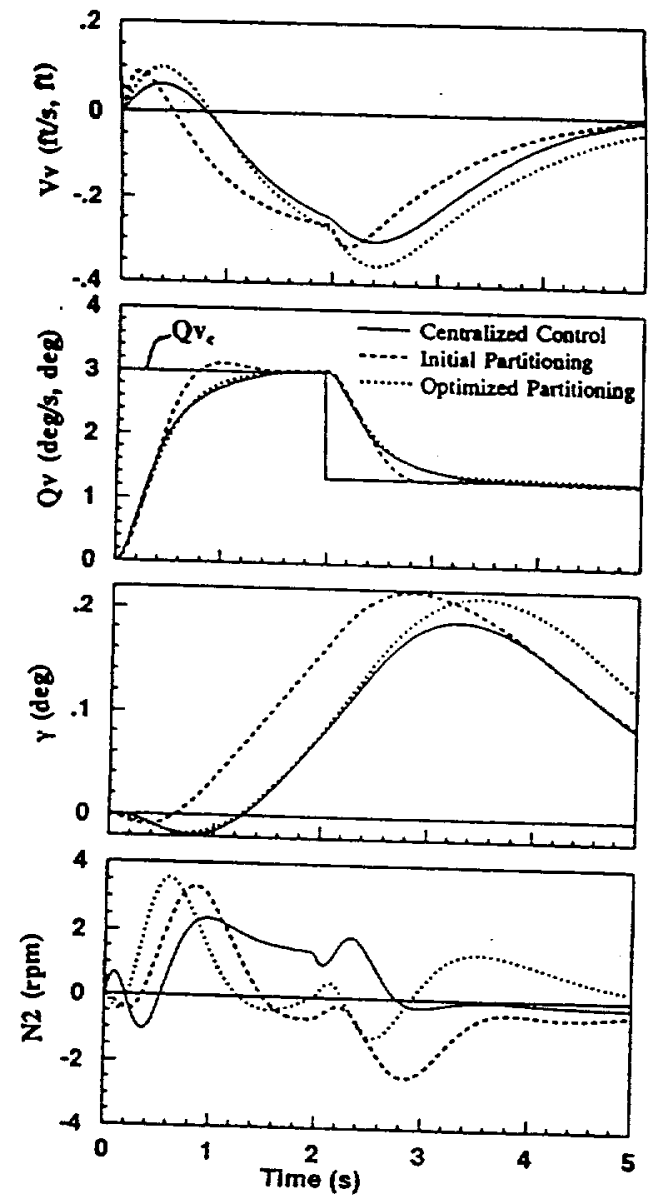

Fig. 4 Closed-loop system response of controlled variables $(\bar{z})$ to $Q v_{c}$

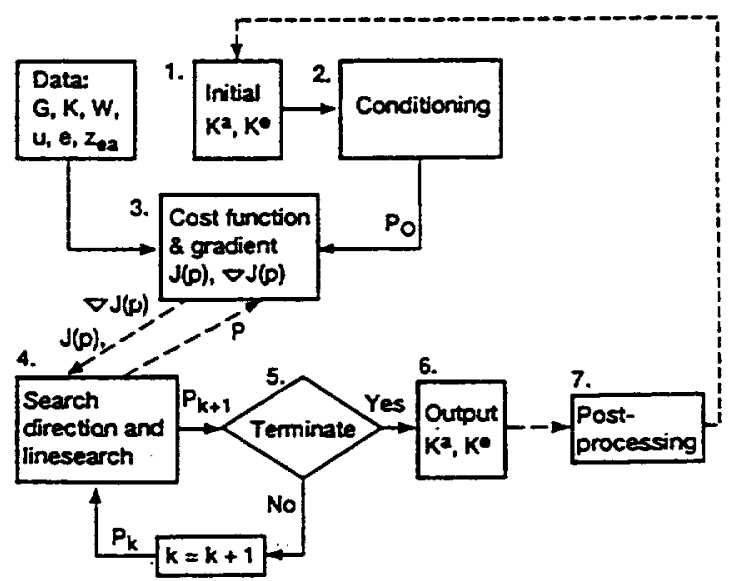

Fig. 2 Flowchart for partitioning optimization algorithm

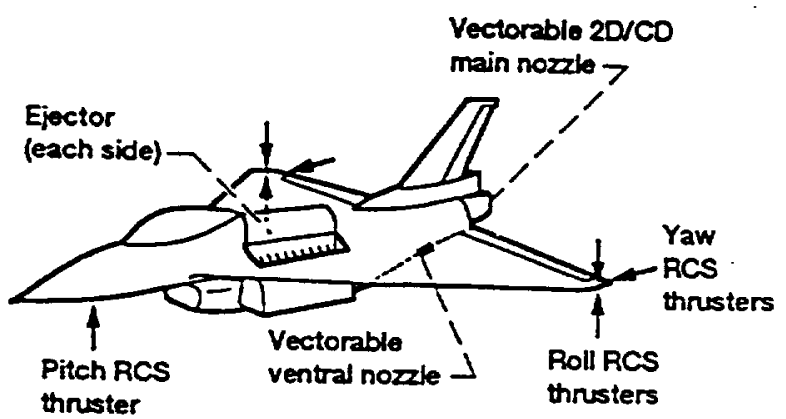

Fig. 3 Schematic of E-TD aircraft showing various control effectors
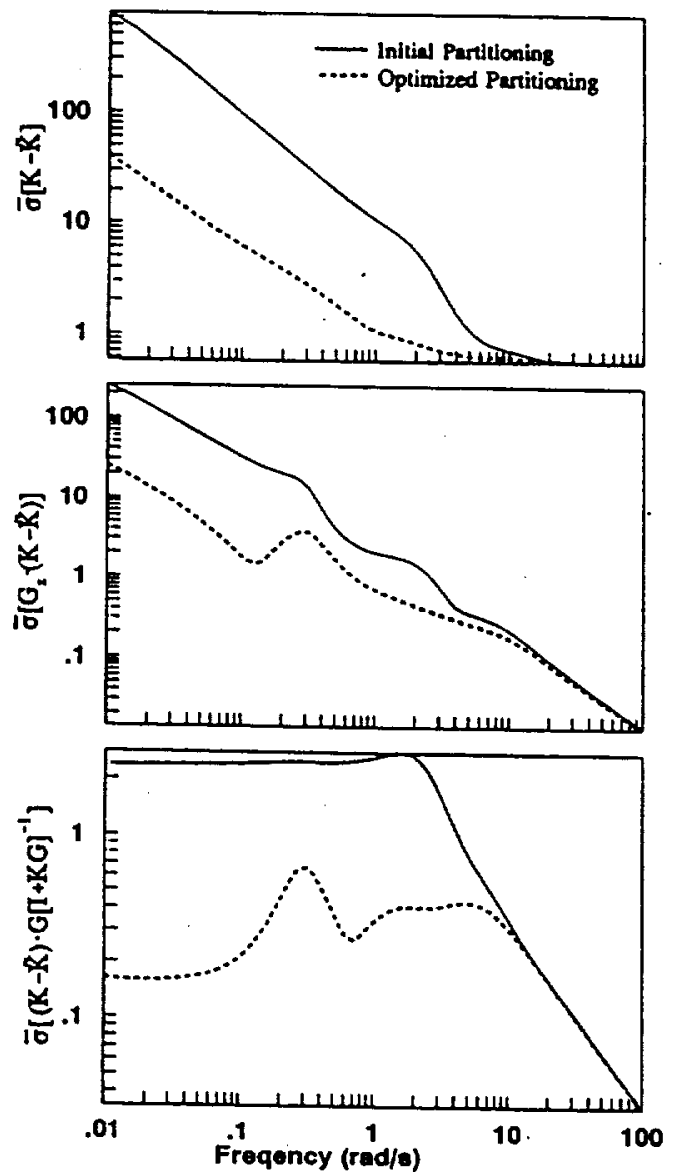

Fig. 5 Maximum singular value of various error measures for partitioned subcontrollers 

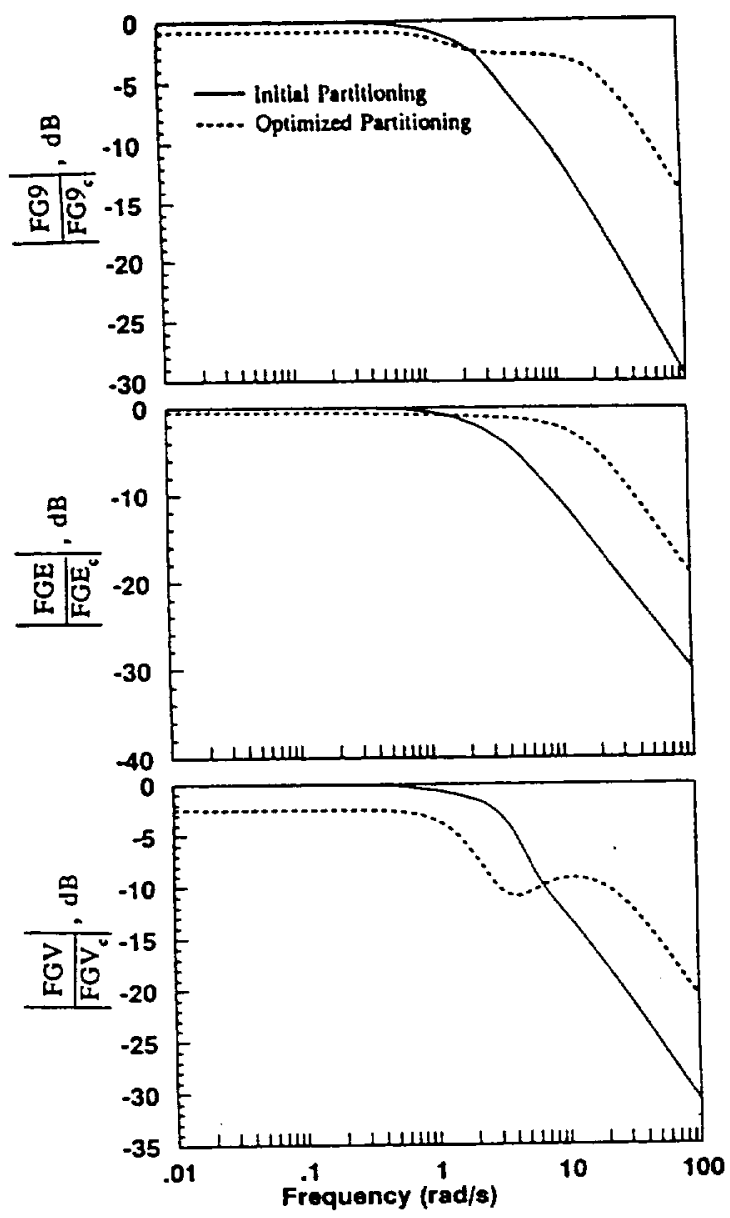

Fig. 6 Bode magnitude plots of $z^{i} \rightarrow z_{a}^{i}$ response for engine closed-loop system with partitioned subcontrollers
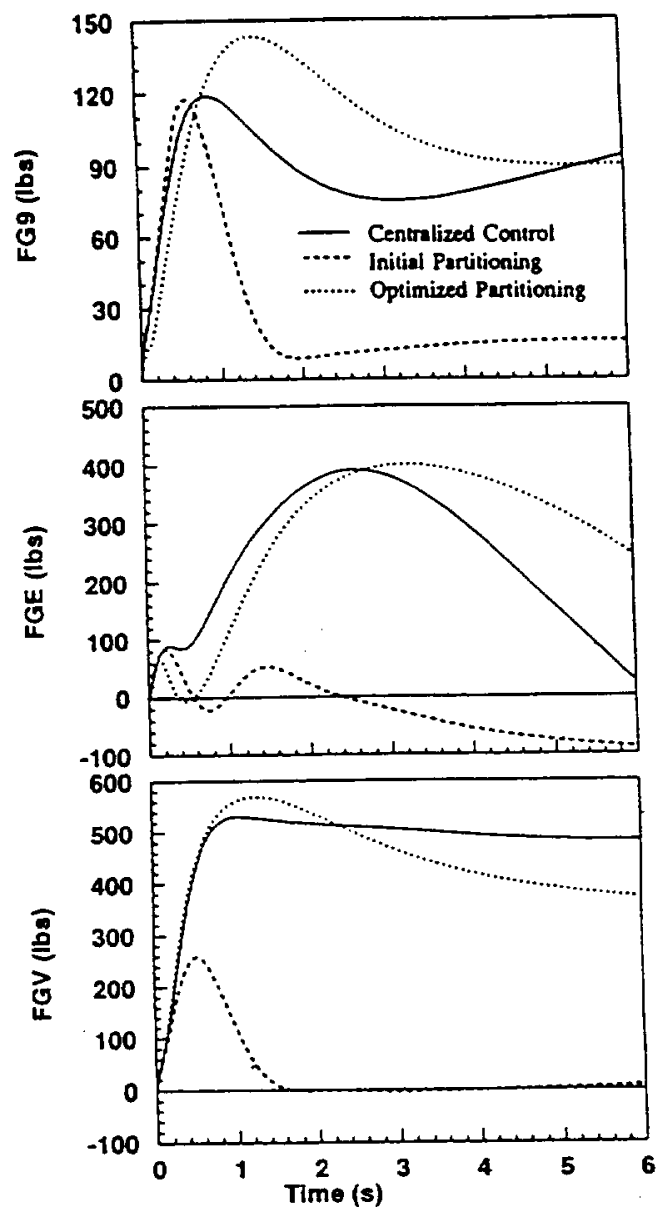

Fig. 7 Closed-loop response of interface variables $\left(\bar{z}_{e}=[\mathrm{FG} 9, \mathrm{FGE}, \mathrm{FGV}]\right)$ to step engine command input $\left(\bar{z}_{e}=N 2_{c}=200 \mathrm{rpm}\right)$ 
Public reporting burden for this collection of information is estimated to average 1 hour per response, including the time for reviewing instructions, searching existing data sources, gathering and maintaining the data needed, and completing and reviewing the collection of information. Send comments regarding this burden estimate or any other aspect of this collection of information, including suggestions for reducing this burden, to Washington Hescquarters Services, Directorate for Intormation Operations and Davis Highway, Suite 1204, Artington, VA 22202-4302, and to the Otfice of Management and Budget, Paperwork Reduction Project (0704-0188), Washington, DC 20503.

\begin{tabular}{|l|c|c|}
\hline 1. AGENCY USE ONLY (Leave blank) & $\begin{array}{c}\text { 2. REPOAT DATE } \\
\text { July } 1993\end{array}$ & $\begin{array}{r}\text { 3. REPORT TYPE AND DATES COVERED } \\
\text { Technical Memorandum }\end{array}$ \\
\hline
\end{tabular}

\section{TILE AND SUBTTLE}

Application of Controller Partitioning Optimization Procedure to Integrated Flight/Propulsion Control Design for a STOVL Aircraft

\section{AUTHOR(S)}

Sanjay Garg and Phillip H. Schmidt
5. FUNDING NUMBERS

WU-505-62-50

\section{PERFORMING ORGANIZATION NAME(S) AND ADDRESS(ES)}

National Aeronautics and Space Administration

Lewis Research Center

Cleveland, Ohio 44135-3191

\section{SPONSORING/MONTORING AGENCY NAME(S) AND ADDRESS(ES)}

National Aeronautics and Space Administration

Washington, D.C. 20546-0001 a. Performing organization REPORT NUMBER

E-7992
10. SPONSORINGMONTORING AGENCY REPORT NUMBER

NASA TM-106265

AIAA-93-3766

\section{SUPPLEMENTARY NOTES}

Prepared for the 1993 AlAA Guidance, Navigation, and Control Conference, sponsored by the American Institute of Aeronautics and Astronautics, Monterey, California, August 9-11, 1993. Sanjay Garg, NASA Lewis Research Center; and Phillip H. Schmidt, University of Akron, Akron, Ohio 44325. Responsible person, Sanjay Garg, (216) 433-2355.

12a. DISTRIBUTION/AYALABULTY STATEMENT | 12b. DISTRIBUTION CODE

Unclassified - Unlimited

Subject Categories 08 and 63

13. ABStTACT (Maximum 200 words)

A parameter optimization framework has earlier been developed to solve the problem of partitioning a centralized controller into a decentralized, hierarchical structure suitable for integrated flight/propulsion control implementation. This paper presents results from the application of the controller partitioning optimization procedure to IFPC design for a Short Take-Off and Vertical Landing (STOVL) aircraft in transition flight. The controller partitioning problem and the parameter optimization algorithm are briefly described. Insight is provided into choosing various "user" selected parameters in the optimization cost function such that the resulting optimized subcontrollers will meet the characteristics of the centralized controller that are crucial to achieving the desired closed-loop performance and robustness, while maintaining the desired subcontroller structure constraints that are crucial for IFPC implementation. The optimization procedure is shown to improve upon the initial partitioned subcontrollers and lead to performance comparable to that achieved with the centralized controller. This application also provides insight into the issues that should be addressed at the centralized control design level in order to obtain implementable partitioned subcontrollers.

\section{SUBJECT TERMS}

Integrated control; Propulsion control; Flight control; Centralized control; Decentralized control

17. SECURITY CLASSIFICATION OF REPORT

Unclassified
18. SECURITY CLASSIFICATION OF THIS PAGE Unclassified
19. SECUATY CLASSIFICATION OF ABSTRACT

Unclassified 